\title{
Article
}

\section{Zolmitriptan Intranasal Spanlastics for Enhanced Migraine Treatment; Formulation Parameters Optimized via Quality by Design Approach}

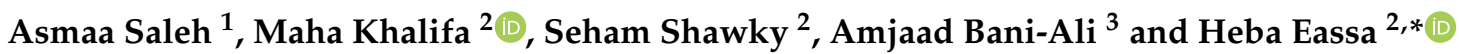 \\ 1 Department of Pharmaceutical Sciences, College of Pharmacy, Princess Nourah bint Abdulrahman University, \\ Riyadh 11671, Saudi Arabia; ASAli@pnu.edu.sa \\ 2 Department of Pharmaceutics and Industrial Pharmacy, Faculty of Pharmacy, Al-Azhar University, \\ Cairo 11651, Egypt; mahakhalifa.pharmg@azhar.edu.eg (M.K.); sehamshawky@azhar.edu.eg (S.S.) \\ 3 Pharmacy Practice Department, College of Pharmacy, Princess Nourah bint Abdulrahman University, \\ Riyadh 11671, Saudi Arabia; amjaad.abd@hotmail.com \\ * Correspondence: hebaabdullah@azhar.edu.eg; Tel.: +20-114-261-2925
}

check for

updates

Citation: Saleh, A.; Khalifa, M.; Shawky, S.; Bani-Ali, A.; Eassa, H. Zolmitriptan Intranasal Spanlastics for Enhanced Migraine Treatment; Formulation Parameters Optimized via Quality by Design Approach. Sci. Pharm. 2021, 89, 24. https://doi.org/ $10.3390 /$ scipharm 89020024

Academic Editor: William A. Donaldson

Received: 9 April 2021

Accepted: 25 May 2021

Published: 27 May 2021

Publisher's Note: MDPI stays neutral with regard to jurisdictional claims in published maps and institutional affiliations.

Copyright: (c) 2021 by the authors. Licensee MDPI, Basel, Switzerland. This article is an open access article distributed under the terms and conditions of the Creative Commons Attribution (CC BY) license (https:// creativecommons.org/licenses/by/ $4.0 /)$.

\begin{abstract}
Zolmitriptan is a potent second-generation triptan prescribed for migraine attacks. It suffers low bioavailability ( $40 \%$ ) after oral administration due to the hepatic first-pass metabolism. Spanlastics are surfactant-based elastic vesicular drug carrier systems. This study aimed to design and optimize intranasal spanlastic formulations as an alternative approach that directly targets brain delivery, enhancing its bioavailability and avoiding the first-pass effect. The quality by design approach was applied to correlate the formulation parameters (Span 60 and Tween 80 concentrations) and critical quality attributes (entrapment efficiency (EE\%) and particle size). Spanlastic formulations were designed based on response surface central composite design and prepared via an ethanol injection method. Designed formulations were characterized by EE\% and particle size measurements to select the optimized formula (with a combination of small particle size and high EE\%). The optimized formula was further subjected to transmission electron microscopy, zeta potential measurement and ex vivo permeation study. The optimized formulation showed a particle size of $117.5 \mathrm{~nm}$ and $\mathrm{EE} \%$ of $45.65 \%$, with a low percentage of error between the observed and predicted values. Seventy percent of zolmitriptan was permeated through the nasal membrane within $30 \mathrm{~min}$, and it completely permeated within $2 \mathrm{~h}$ with a significantly higher steady-state flux compared to plain gel. This study introduced a successful and promising intranasal formulation suitable for further brain delivery analysis.
\end{abstract}

Keywords: zolmitriptan; intranasal brain delivery; quality by design; ex vivo permeation

\section{Introduction}

Migraines are a neurovascular disorder characterized by severe headache and trigeminovascular system activation involving the release of calcitonin-gene-related peptide (CGRP) [1]. It is characterized by being throbbing and unilateral and is usually accompanied by nausea and sensitivity to sound and light [2]. The exact pathophysiology underlying migraine headaches is still not fully understood. For a long period, the vascular origin of migraines was adopted, relating the headache to prolonged vasodilatation of cranial blood vessels [2]. However, abundant evidence in the past decades suggests an integrated theory of vascular and neuronal components. The neurogenic inflammation theory involves the release of vasoactive neuropeptides (CGRP, substance P (SP) and neurokinin A), causing a cascade of inflammatory tissue responses [3].

Treatment usually includes NSAID, ergot alkaloids and 5-HT receptor agonist (trip$\tan$ ) [4]. Triptans including zolmitriptan are $5 \mathrm{HT1B} / 1 \mathrm{D}$ serotonin receptor agonists and considered the forefront in migraine treatment [2,5]. Triptans attenuate the CGRP levels 
evoked by electrical stimulation of the trigeminal ganglion and superior sagittal sinus [6]. The agonistic action on $5 \mathrm{HT} 1 \mathrm{~B} / 1 \mathrm{D}$ receptors inhibits the release of vasoactive neuropeptides by trigeminal nerves and inhibits nociceptive neurotransmission along with vasoconstriction of painfully dilated cerebral blood vessels [7]. Zolmitriptan reduces migraine symptoms efficiently and therefore is considered an excellent therapy [8,9]. However, the slow onset of action and poor bioavailability (40\%) due to the hepatic first-pass metabolism are the major drawbacks of zolmitriptan oral formulations [8]. Zolmitriptan has a half-life of 1-2 h [10]. Moreover, migraines are accompanied with gastric stasis and vomiting, which may cause delayed or inconsistent absorption of medications [11]. A route which improves the bioavailability, enhances brain targeting and avoids the first-pass metabolism would be highly adequate for zolmitriptan delivery.

The central nervous system (CNS) is highly protected by two barriers: the blood-brain barrier (BBB) and the blood-cerebrospinal fluid barrier. The BBB is a physical as well as biochemical barrier. It expresses enzymes and efflux p-glycoprotein, and therefore it is the rate-limiting factor that determines drug permeation to the brain [12,13]. Thus, brain drug delivery is challenging [14]. The intranasal route can directly target the central nervous system [15]. It is one of the strategies utilized to transport drugs to the brain via different pathways [16]. Moreover, the intranasal route can reduce the possible side effects and increase the bioavailability of drugs by avoiding the hepatic first-pass metabolism [17].

Since 1995, the use of nanoparticles for systemic delivery of therapeutics has increased. Size, shape and flexibility are essential physical parameters that influence nanoparticles' penetration and transport across the BBB [18]. Spanlastics are surfactant-based nanovesicular systems that are modified from niosomes by the addition of edge activators [19]. Spanlastics are formed by a Span ${ }^{\circledR}$ (Surface active agent) and an edge activator (Tween 80). Spanlastics can be used to deliver both hydrophilic and hydrophobic drugs, which are encapsulated by the interior hydrophilic compartment and the outer lipid layer, respectively [1]. Spanlastic formulations are capable of transporting hydrophilic drugs such as zolmitriptan across the BBB, and this capability is based on the spanlastic characteristics [20]. For instance, edge activators impart elasticity to these systems, which allow the transport of the vesicles through membrane barriers [21]. Abdelmonem et al. formulated a granisetron hydrochloride spanlastic in mucoadhesive gels and lyophilized inserts for intranasal administration to improve drug bioavailability and brain targeting [16]. Moreover, spanlastics have been utilized for targeting topical drug(s) to the posterior segment of the eye [21]. Yassin et al. used spanlastics for more efficient targeting of carbamazepine to the brain via the intranasal route [22].

Quality by design (QbD) is a novel approach that has been adopted by the US FDA. It is a systematic approach for designing the final product based on certain quality requirements known as critical quality attributes (CQAs) of the formulation [23]. Application of QbD assures the final product quality through enhanced understating and implementation of both process and formulation parameters [24].

In this study, intranasal spanlastic formulations were designed using the QbD approach to investigate the correlation between formulation parameters (Span 60 and Tween 80 concentrations) and CQAs (entrapment efficiency and particle size) and optimize a formulation suitable for intranasal drug delivery to the brain [16].

\section{Materials and Methods}

\subsection{Materials}

Zolmitriptan was kindly provided by Hikma Pharmaceuticals, Cairo, Egypt. Span 60 and Tween 80 were purchased from Sigma-Aldrich, St. Louis, USA. All other materials were of analytical grade.

\subsection{Preparation of Zolmitriptan-Loaded Spanlastic (ZLS) Formulations}

Spanlastics were formulated through an ethanol injection method [16]. Briefly, $10 \mathrm{~mL}$ absolute ethanol were used to dissolve zolmitriptan and Span 60. The alcoholic solution 
was warmed and injected slowly into a preheated $\left(70{ }^{\circ} \mathrm{C}\right)$ aqueous solution $(10 \mathrm{~mL})$ in which Tween 80 (edge activator) was previously dissolved. Stirring was continued on a magnetic stirrer for $1 \mathrm{~h}$ until complete evaporation of the ethanol. The formed dispersion was sonicated for 2 min to develop fine spanlastic dispersion and prevent aggregates from forming. Finally, the formulations were preserved in a refrigerator until being investigated.

\subsection{Statistic Optimization of Spanlastic Formulations}

The Design of the Experiment

Based on previous research, we chose the concentrations of Span 60 and Tween 80 to be the formulation parameters (independent variables, $\mathrm{X} 1$ and $\mathrm{X} 2$, respectively). Their effects on the CQAs of the developed formulation, namely entrapment efficiency (Y1) and particle size (Y2), were investigated using a 2-factor, 3-level DoE (Table 1). Thirteen formulations were prepared based on a response surface central composite design (Design-Expert $R$ software version 13 state-ease), and their CQAs were characterized, as shown in Table 2.

Table 1. Experimental range of formulation parameters (factors) and critical quality attributes (CQAs) of zolmitriptan-loaded spanlastics.

\begin{tabular}{cccc}
\hline Formulation & \multicolumn{2}{c}{ Levels } \\
\cline { 2 - 4 } Parameters (Factors) & $\mathbf{- 1}$ & $\mathbf{0}$ & $\mathbf{1}$ \\
\hline $\begin{array}{c}\text { X1: Span 60 } \\
\text { concentration }\end{array}$ & $65 \mathrm{mg}$ & $75 \mathrm{mg} \mathrm{mg}$ \\
\hline $\begin{array}{c}\text { X2: Tween } 80 \\
\text { concentration }\end{array}$ & $15 \mathrm{mg}$ & $25 \mathrm{mg}$ & $35 \mathrm{mg}$ \\
\hline Response (CQAs) & & constraints & \\
\hline $\begin{array}{c}\text { Y1: Entrapment } \\
\text { efficiency }\end{array}$ & maximize \\
\hline Y2: Particle size & minimize \\
\hline
\end{tabular}

Table 2. Experimental runs, formulation parameters (described in coded levels) and critical quality attributes (CQAs) of zolmitriptan-loaded spanlastics.

\begin{tabular}{ccccc}
\hline \multicolumn{2}{c}{ Formulation Parameters } & \multicolumn{2}{c}{ CQAs (Responses) } \\
\hline Run & $\begin{array}{c}\text { X1: Span 60 } \\
\text { Concentration }\end{array}$ & $\begin{array}{c}\text { X2: Tween 80 } \\
\text { Concentration }\end{array}$ & $\begin{array}{c}\text { Y1: Entrapment } \\
\text { Efficiency } \\
\mathbf{( \% )}\end{array}$ & $\begin{array}{c}\text { Y2: Particle Size } \\
\text { (nm) }\end{array}$ \\
\hline 1 & 0 & -1.41421 & 131.2 & 53.42 \\
2 & 0 & 0 & 96.12 & 48.32 \\
3 & -1 & 1 & 40.75 & 20.7 \\
4 & 0 & 1.41421 & 90.17 & 40.21 \\
5 & 0 & 0 & 96.12 & 48.32 \\
6 & 0 & 0 & 96.12 & 48.32 \\
7 & -1 & -1 & 21.07 & 55.21 \\
8 & 0 & 0 & 96.12 & 48.32 \\
9 & 1.41421 & 0 & 172.3 & 61.92 \\
10 & -1.41421 & 0 & 60.73 & 23.22 \\
11 & 1 & -1 & 175.24 & 63.47 \\
12 & 0 & 0 & 96.12 & 48.32 \\
13 & 1 & 1 & 168.4 & 57.98 \\
\hline
\end{tabular}

\subsection{Characterization of Zolmitriptan-Loaded Spanlastic (ZLS) Formulations}

2.4.1. Entrapment Efficiency Percentage (EE\%) Determination

The supernatant containing non-encapsulated zolmitriptan was obtained by subjecting samples from each formula to centrifugation using a cooling centrifuge (Beckman, 
Fullerton, Canada) at 15,000 rpm for $30 \mathrm{~min}$. The clear supernatant was collected using a micropipette. The concentration of the free drug fraction (unentrapped drug) was determined spectrophotometrically (UV-1601 PC spectrophotometer, Shimadzu, Kyoto, Japan) at $282 \mathrm{~nm}$ [6]. The quantity of zolmitriptan entrapped in each formula was estimated according to this equation [24]:

$$
\mathrm{EE} \%=\frac{\text { Total amount of drug }- \text { Amount of unentrapped drug }}{\text { Total amount of drug }} \times 100
$$

\subsubsection{Particle Size Analysis}

The analysis of ZLS particle size was performed via a Zeta-sizer 3000 PCS (Malvern Instr. Ltd., Malvern WR14 1XZ, England). Prior to the measurements, samples were appropriately diluted with double-distilled water. The averages of 3 measurements over 5 min each are given.

\subsection{Design Space Optimization and Model Validation}

The desired criteria were set for CQAs, and the design space was generated accordingly. Then, the optimized formula was prepared and evaluated to compare its CQAs with the predicted values. The model was validated by calculating the percentage error. Further characterization was performed for the optimized formulation.

\subsection{Optimized Formula Characterization}

\subsubsection{Entrapment Efficiency Percentage Determination}

The sample was diluted properly prior to the measurements and transferred to the quartz cuvette of the Zeta-sizer (Zeta-sizer 3000 PCS (Malvern Instr. Ltd., Malvern WR14 1XZ England)) for measurement of the particle size, PDI and ZP.

2.6.2. Determination of Particle Size, Polydispersity Index (PDI) and Zeta Potential (ZP)

The sample was diluted properly prior to the measurements and transferred to the quartz cuvette of the Zeta-sizer (Zeta-sizer 3000 PCS (Malvern Instr., England)) for measurement of the particle size, PDI and ZP.

\subsubsection{Transmission Electron Microscope (TEM) Imaging}

A 1:1000 dilution with water was done for the tested sample. A drop of the sample was allowed to settle on a mesh carbon-coated copper grid for $35 \mathrm{~min}$. An absorbent paper was used to remove excess fluid. The sample was stained with uranyl acetate stain and examined by TEM (JEM 1010, JEOL Ltd., Tokyo, Japan) with an acceleration voltage of $70 \mathrm{kV}$ and magnification power of $50,000 \times$.

\subsection{Preparation of Zolmitriptan-Loaded Spanlastic (ZLS) Nasal Gel}

A cooling centrifuge was used to remove the unentrapped drug of the ZLS dispersion. The residue of ZLS was then dispersed in a gel base ( $2 \%$ methyl cellulose) and stirred until a homogenous gel was obtained. Meanwhile, a control gel for comparison purpose was prepared by dissolving $2.5 \mathrm{mg}$ of zolmitriptan in $2 \%$ methyl cellulose gel, and both were kept in a refrigerator [16].

\subsection{Ex Vivo Permeation}

Zolmitriptan permeation from spanlastic gel formulation through sheep nasal membrane was investigated and compared with its permeation from the control gel formulation. Nasal membranes were excised from sheep nostril. The excised mucosal membranes were frozen at $-80{ }^{\circ} \mathrm{C}$ in phosphate buffer saline ( $\mathrm{pH}$ 7.4). The outer tube of a plastic syringe (10 mL capacity) was cut smoothly to the hole diameter near the nozzle and was used as the donor compartment. The mucosal membrane was stretched around the cut end of the syringe, giving a permeation area of $3.14 \mathrm{~cm}^{2}$. An accurately weighed amount of 
spanlastic gel formulation was introduced into the syringe attached to the shafts of the USP I dissolution apparatus rotating at $50 \mathrm{rpm}$. Phosphate buffer solution ( $\mathrm{pH} 7.4$ ) was utilized as the dissolution medium, placed in the receptor compartment at $37^{\circ} \mathrm{C}$. Adequate samples $(3 \mathrm{~mL})$ were withdrawn during the release, testing at certain time intervals. The drug concentration was estimated spectrophotometrically at $\lambda \max 282 \mathrm{~nm}$ [6]. The experiment was carried out in triplicate.

To estimate the steady state flux Jss, the amount of zolmitriptan permeated across $1 \mathrm{~cm}^{2}$ of the nasal membrane over time was estimated according to the equation [25]:

$$
\mathrm{Jss}=\Delta \mathrm{Qt} /(\Delta \mathrm{t} \times \mathrm{S})
$$

where Qt is the cumulative amount of zolmitriptan permeated and $S$ is the surface area of the membrane [25].

\section{Results and Discussion}

\subsection{Influence of Formulation Factors on CQAs}

Zolmitriptan spanlastics were formulated via an ethanol injection method. Span 60 was chosen as it allows unilamellar as well as multilamellar vesicles and provides sustainability to these developed vesicles. Moreover, it can synergize the reducing effect of the edge activator on the interfacial tension, resulting in fine spanlastic dispersion [26]. The experiment was designed based on two factors (formulation parameters) and three levels of each factor. A central composite design was chosen to reduce the estimated variance over the design space $[27,28]$. The experimental levels of Span 60 were established as $65-85$ $\mathrm{mg}$, while the levels of edge activator (Tween 80 ) were established as 15-35 mg (Table 1).

\subsubsection{Influence of Formulation Parameters on Entrapment Efficiency Percentage}

The entrapment efficiency of the ZLS formulations ranged from $20.7 \%$ to $63.47 \%$, as shown in Table 2 . The data were analyzed utilizing polynomial analysis fitted with a quadratic model $(p<0.0001)$. The predicted $\mathrm{R}^{2}(0.8992)$ was in reasonable agreement with the adjusted $R^{2}(0.990)$, indicating good correlation. The polynomial equation that demonstrates the relation between $\mathrm{EE}$ and independent formulation factors is as follows:

Entrapment efficiency \% $(y 1)=48.32+14.58 X 1-5.29 \times 2+3.16 X 1 X 2-2.74 X 12-0.61 X 22$

From the equation, it is clear that EE is positively affected by the Span 60 concentration and negatively affected by the Tween 80 concentration (Figure $1 \mathrm{a}, \mathrm{b})$. ANOVA results indicate significant effects of both factors on the EE\% of ZLS $(p>0.0001)$. The increase in Span 60 concentration (X1) significantly increased EE\% at $p<0.0001$, which could be explained by the solid nature, hydrophobicity and high phase transition temperature $\left(\mathrm{T}^{\circ} \mathrm{C}\right)$ $\left(53{ }^{\circ} \mathrm{C}\right)$ of Span 60 [29]. Al-Mahallawi et al. related this to the highly saturated alkyl chain and phase transition temperature $\left(\mathrm{T}^{\circ} \mathrm{C}\right)$ of Span 60 , which influenced drug EE\% in bilosomes [30]. However, increasing the Tween 80 concertation significantly reduced $\mathrm{EE} \%(p<0.0001)$. The unsaturation of Tween 80 alkyl chains allows it to bend, increasing the spanlastic's permeability. Therefore, the probability of drug leakage increases [31]. Based on this, the highest EE\% can be obtained by increasing the Span 60 concertation and decreasing the Tween 80 concentration. Moreover, Span 60 has HLB $=4.7$ and Tween 80 has HLB $=15$. The combination of a high Span 60 concertation and a low Tween 80 concentration results in a slightly lipophilic HLB value. Zolmitriptan, being lipophilic, would favorably be entrapped in a lipophilic spanlastic matrix [31]. 


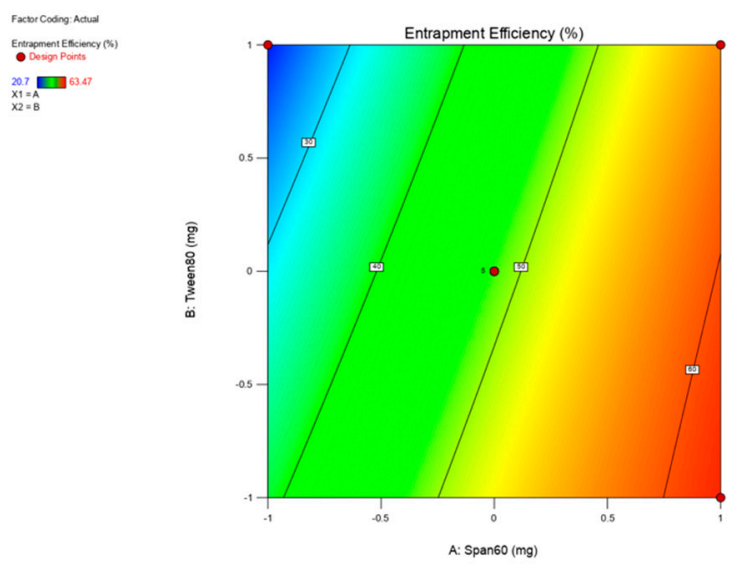

(a)

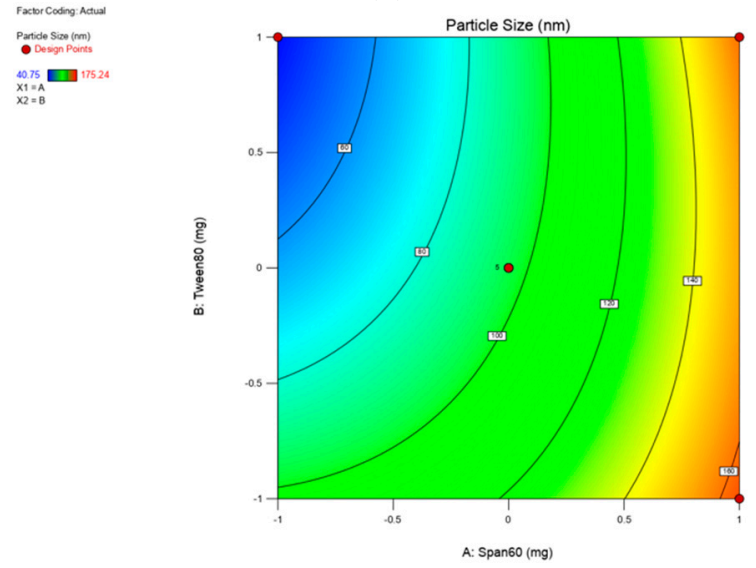

(c)

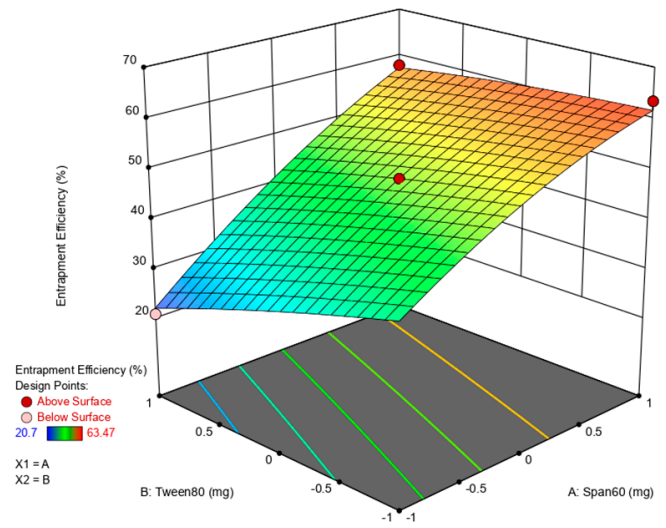

(b)

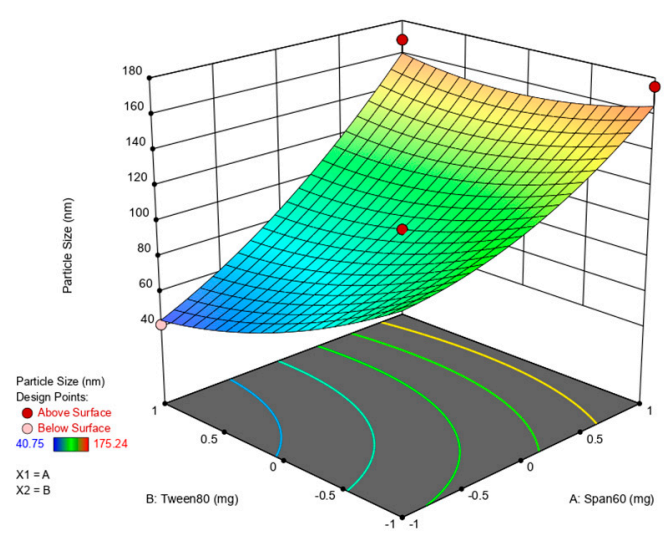

(d)

Figure 1. Contour and 3D-surface plots for the effect of Span 60 and Tween 80 concentrations on the following for zolmitriptan spanlastics formulations: entrapment efficiency $(\mathbf{a}, \mathbf{b})$; and particle size $(\mathbf{c}, \mathbf{d})$.

\subsubsection{Influence of Formulation Parameters on Particle Size}

It has clearly been demonstrated that the intracellular uptake and, consequently, brain targeting are related to particle size [32]. The particle sizes of ZLS formulations ranged from 40.75 to $175.24 \mathrm{~nm}$, as shown in Table 2. The obtained data were analyzed utilizing polynomial analysis fitted with a quadratic model, resulting in $p<0.0001$, which indicates the significance of the model.

The polynomial equation that demonstrates the relation between particle size and independent formulation factors is as follows:

$$
\text { Particle size }(\mathrm{y} 2)=96.12+44.79 \times 1-15.81 \mathrm{X} 2+13.69 \times 1 \times 2+12.22 \times 12+9.30 \times 22
$$

It can be concluded that increasing the Span 60 concentration significantly increases the particle size $(p<0.0001)$, as shown in Figure 1c,d. Additionally, the Tween 80 concentration significantly decreased the particle size $(p<0.002)$. This is due to the unsaturation of the alkyl chain, allowing chain bending and results in a smaller size. Additionally, a high Tween 80 concentration reduces the interfacial tension, causing particle portioning [33].

Moreover, based on the low HLB of the system, smaller vesicles are produced due to decreases in both surface energy and water uptake along with increased hydrophobicity [34]. 


\subsection{Optimized Formula Characterization}

3.2.1. Entrapment Efficiency, Particle Size, Zeta Potential and Polydispersity Index (PDI) of the Optimized Formula

The optimized formula was selected based on the response surface analysis of the central composite design and contained $74.3 \mathrm{mg}$ of Span 60 and $23.005 \mathrm{mg}$ of Tween 80 . It was predicted to have a particle size of $94.59 \mathrm{~nm}$ and $\mathrm{EE} \%$ of 47.77 . The optimized formula was evaluated and compared to the predicted values. The optimized formula showed vesicles of $117.5 \mathrm{~nm}$ size and EE\% of $45.65 \%$. It showed percent errors of $0.23 \%$ and $-0.021 \%$ for particle size and $\mathrm{EE} \%$, respectively. This low magnitude of error indicates that the central composite model is adequate and provides high prediction in optimizing spanlastic formulations [24].

Moreover, the optimized formula showed a PDI of 0.685 and a ZP of $-28.0 \mathrm{mV}$. The PDI value suggests the reasonable homogeneity of the formulation [33]. Zeta potential value predicts vesicular stability [35]. The high value of $Z P$ indicates a good repulsion between charged particles, which electrically stabilizes the colloidal particles and protects them from reaggregation [36]. In addition, the negative charge of the vesicles is a result of the hydroxyl groups of the non-ionic surfactants [37].

\subsubsection{Transmission Electron Microscope (TEM) Imaging}

The morphology of the optimized formula was examined through TEM technique, as presented in Figure 2. The optimized formula showed spherical and uniform shape without aggregation. Vesicles have a well-defined spherical shape with defined boundaries, which is related to the amphiphilic nature of the non-ionic surfactants. Non-ionic surfactants tend to develop spherical vesicles, which decrease the surface-free energy [33].

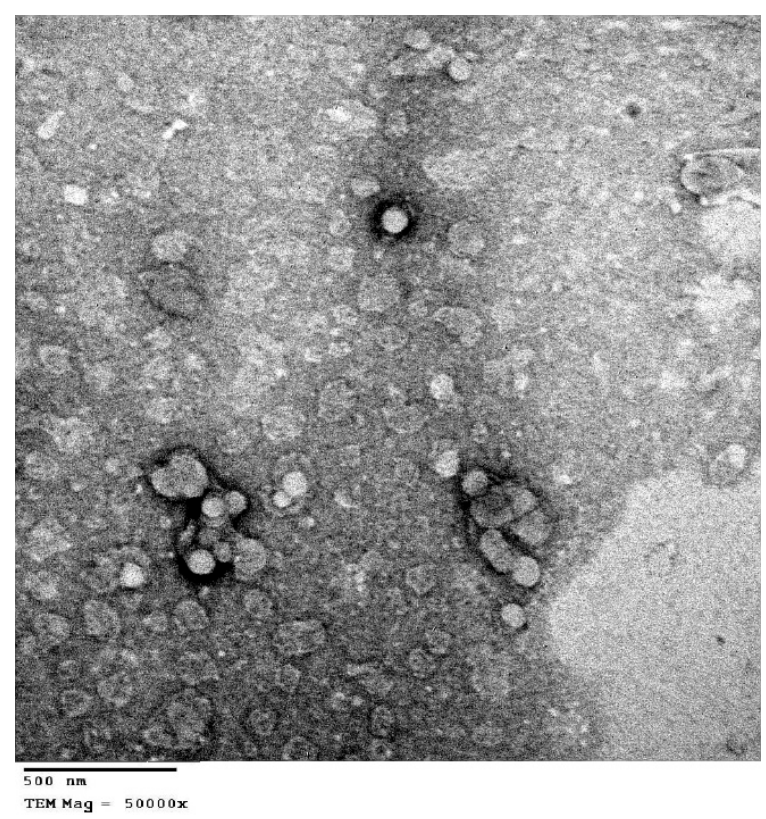

Figure 2. Transmission electron micrograph of the optimized formula of the zolmitriptan-loaded spanlastic.

The particles size was within $100 \mathrm{~nm}$, which confirmed the values obtained by PS measurements. Previous studies have reported that the size, shape and charge of nanoparticles influence their in vivo biological performance [38]. Particles with smaller size have been reported to escape clearance by the reticuloendothelial system (RES) [38]. The small particle size and shape confirmed by TEM ensure the ability of these particles to achieve longer residence in the systemic circulation via bypassing uptake by RES. Moreover, Tween 80 was found to deceases RES clearance and facilitate the brain uptake [39]. 


\subsubsection{Ex Vivo Permeation}

Zolmitriptan permeation via the nasal membrane was an important parameter to ensure the privilege of the spanlastic formulation over the plain gel formulation in vivo. Both formulations were best fitted by the Baker and Lansodal equation, with $\mathrm{r}^{2}$ values of 0.966 and 0.906 for the optimized formulation and the zolmitriptan gel formulation, respectively. The results show that the zolmitriptan permeation rate from the spanlastic formula increased significantly when compared to its permeation rate from the plain control gel formulation, as demonstrated in Figure 3. It was clearly observed that $70 \%$ of zolmitriptan was permeated through the nasal membrane in the first $30 \mathrm{~min}$, and it completely permeated within $2 \mathrm{~h}$. However, in the case of the gel formulation, only $59 \%$ of zolmitriptan was permeated after $2 \mathrm{~h}$. The steady state flux (Jss) of the optimized formula was $48.48 \mu \mathrm{g} / \mathrm{cm}^{2} / \mathrm{h}$, which was significantly higher than the Jss of the plain gel $\left(29.65 \mu \mathrm{g} / \mathrm{cm}^{2} / \mathrm{h}\right)$.

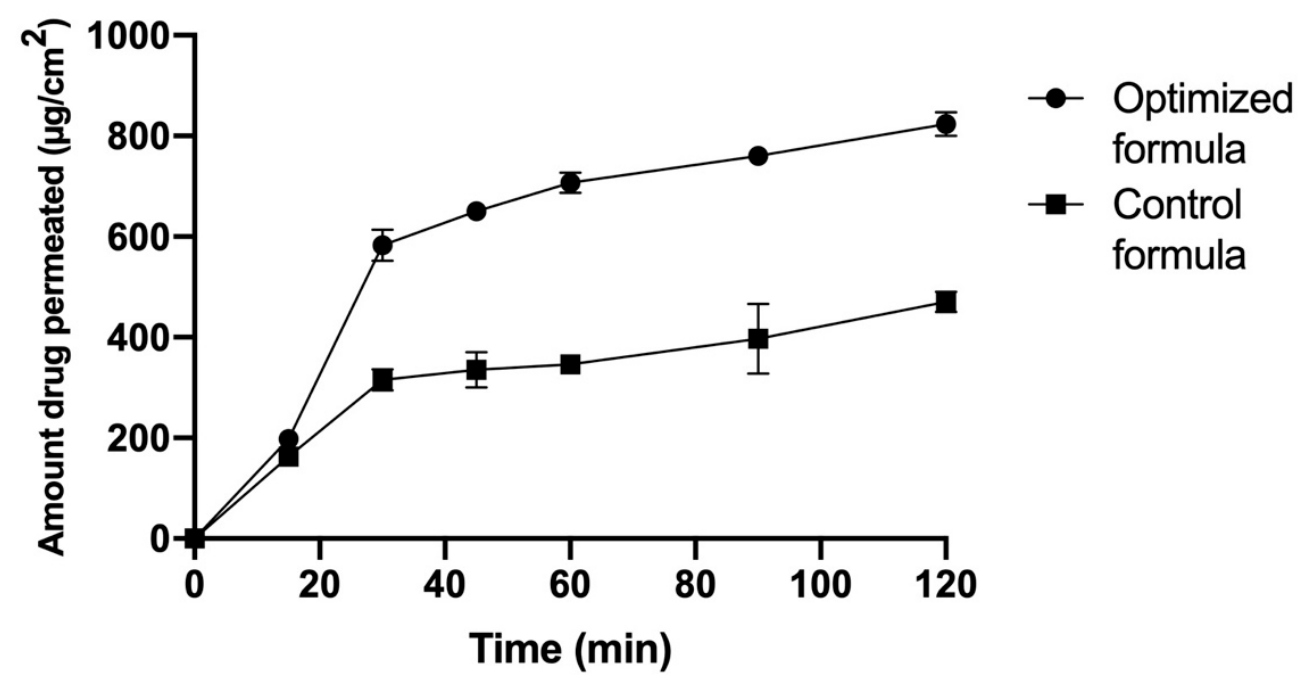

Figure 3. Zolmitriptan ex vivo permeation from the spanlastic optimized formula compared to the control gel formula through the nasal membrane.

This improvement in drug permeation could be explained by the nano-size of the optimized formulation and the presence of surfactants [19]. Tween 80 has been reported to increase the permeation across biological membranes such as the nasal membrane and BBB [40]. Spanlastic content in surfactants has been reported to facilitate vesicle squeezing intercellularly and their permeation due to the elastic effect [41]. This elasticity improves the skin penetration capability of the vesicles. Moreover, the vesicles maintain their integrity, achieving enhanced delivery of the entrapped drug [42]. Additionally, permeation with better interaction with the skin layers could be related to Span 60 vesicles, due to the low HLB value $(\mathrm{HLB}=4.7)[43]$.

\section{Conclusions}

In this study, the QbD approach was applied to comprehend the influence of formulation parameters on the characteristics of zolmitriptan-loaded spanlastic formulations and successfully predicted the optimized formulation. The optimized formulation showed enhanced drug permeation through the nasal membrane, confirming the promising effect of intranasal route for targeting brain delivery. Preclinical study can be carried out using the in vivo BBB model to further investigate the in vitro-in vivo correlation.

Author Contributions: Conceptualization, A.S., M.K., S.S. and H.E.; methodology, M.K, S.S. and H.E.; software, H.E.; writing-original draft preparation, A.B.-A., M.K. and H.E.; and writing-review 
and editing, A.S., M.K. and H.E. All authors have read and agreed to the published version of the manuscript.

Funding: This research was funded by the Deanship of Scientific Research at Princess Nourah bint Abdulrahman University through the Fast-track Research Funding Program.

Institutional Review Board Statement: Not applicable.

Informed Consent Statement: Not applicable.

Conflicts of Interest: The authors declare no conflict of interest.

\section{References}

1. De Vries, T.; Villalón, C.M.; MaassenVanDenBrink, A. Pharmacological treatment of migraine: CGRP and 5-HT beyond the triptans. Pharmacol. Ther. 2020, 211, 107528. [CrossRef]

2. Mahmoud, A.A.; Salah, S. Fast relief from migraine attacks using fast-disintegrating sublingual zolmitriptan tablets. Drug Dev. Ind. Pharm. 2012, 38, 762-769. [CrossRef]

3. Malhotra, R. Understanding migraine: Potential role of neurogenic inflammation. Ann. Indian Acad. Neurol. 2016, 19, 175-182. [CrossRef]

4. Poply, K.; Bahra, A.; Mehta, V. Migraine. BJA Educ. 2016, 16, 357-361. [CrossRef]

5. Spierings, E.L.; Brandes, J.L.; Kudrow, D.B.; Weintraub, J.; Schmidt, P.C.; Kellerman, D.J.; Tepper, S.J. Randomized, double-blind, placebo-controlled, parallel-group, multi-center study of the safety and efficacy of ADAM zolmitriptan for the acute treatment of migraine. Cephalalgia 2018, 38, 215-224. [CrossRef] [PubMed]

6. Hargreaves, R.; Olesen, J. Calcitonin Gene-Related Peptide Modulators-The History and Renaissance of a New Migraine Drug Class. Headache J. Head Face Pain 2019, 59, 951-970. [CrossRef] [PubMed]

7. Tepper, S.J.; Rapoport, A.M.; Sheftell, F.D. Mechanisms of Action of the 5-HT1B/1D Receptor Agonists. Arch. Neurol. 2002, 59, 1084-1088. [CrossRef] [PubMed]

8. Prajapati, S.T.; Patel, M.V.; Patel, C.N. Preparation and evaluation of sublingual tablets of zolmitriptan. Int. J. Pharm. Investig. 2014, 4, 27-31. [CrossRef]

9. Saxena, P.R.; Tfelt-Hansen, P. Success and failure of triptans. J. Headache Pain 2001, 2, 3-11. [CrossRef]

10. Mostafa, D.A.E.; Khalifa, M.K.A.; Gad, S.S. Zolmitriptan brain targeting via intranasal route using solid lipid nanoparticles for migraine therapy: Formulation, characterization, in-vitro and in-vivo assessment. Int. J. Appl. Pharm. 2020, 10, 86-93. [CrossRef]

11. Aurora, S.K.; Papapetropoulos, S.; Kori, S.H.; Kedar, A.; Abell, T.L. Gastric stasis in migraineurs: Etiology, characteristics, and clinical and therapeutic implications. Cephalalgia 2013, 33, 408-415. [CrossRef]

12. Meairs, S.; Alonso, A. Ultrasound, microbubbles and the blood-brain barrier. Prog. Biophys. Mol. Biol. 2007, 93, 354-362. [CrossRef]

13. Pailla, S.R.; Talluri, S.; Rangaraj, N.; Ramavath, R.; Challa, V.S.; Doijad, N.; Sampathi, S. Intranasal Zotepine Nanosuspension: Intended for improved brain distribution in rats. DARU J. Pharm. Sci. 2019, 27, 541-556. [CrossRef] [PubMed]

14. Chen, Y.; Liu, L. Modern methods for delivery of drugs across the blood-brain barrier. Adv. Drug Deliv. Rev. 2012, 64, 640-665. [CrossRef] [PubMed]

15. Sonvico, F.; Clementino, A.; Buttini, F.; Colombo, G.; Pescina, S.; Guterres, S.S.; Pohlmann, A.R.; Nicoli, S. Surface-Modified Nanocarriers for Nose-to-Brain Delivery: From Bioadhesion to Targeting. Pharmaceutics 2018, 10, 34. [CrossRef]

16. Abdelmonem, R.; El Nabarawi, M.; Attia, A. Development of novel bioadhesive granisetron hydrochloride spanlastic gel and insert for brain targeting and study their effects on rats. Drug Deliv. 2018, 25, 70-77. [CrossRef] [PubMed]

17. Wu, C.; Li, B.; Zhang, Y.; Chen, T.; Chen, C.; Jiang, W.; Wang, Q.; Chen, T. Intranasal delivery of paeoniflorin nanocrystals for brain targeting. Asian J. Pharm. Sci. 2020, 15, 326-335. [CrossRef]

18. Nowak, M.; Brown, T.D.; Graham, A.; Helgeson, M.E.; Mitragotri, S. Size, shape, and flexibility influence nanoparticle transport across brain endothelium under flow. Bioeng. Transl. Med. 2020, 5, e10153. [CrossRef]

19. Abdelrahman, F.E.; Elsayed, I.; Gad, M.K.; Elshafeey, A.H.; Mohamed, M.I. Response surface optimization, ex vivo and in vivo investigation of nasal spanlastics for bioavailability enhancement and brain targeting of risperidone. Int. J. Pharm. 2017, 530, 1-11. [CrossRef]

20. El-Nabarawy, N.A.; Teaima, M.H.; Helal, D.A. Assessment of spanlastic vesicles of zolmitriptan for treating migraine in rats. Drug Des. Dev. Ther. 2019, 13, 3929. [CrossRef]

21. Kakkar, S.; Kaur, I.P. Spanlastics-A novel nanovesicular carrier system for ocular delivery. Int. J. Pharm. 2011, 413, 202-210. [CrossRef]

22. Yassin, G.E.; Amer, R.I.; Fayez, A.M. Carbamazepine loaded vesicular structures for enhanced brain targeting via intranasal route: Optimization, in vitro evaluation, and in vivo study. Int. J. Appl. Pharm. 2019, 11, 264-274. [CrossRef]

23. Namjoshi, S.; Dabbaghi, M.; Roberts, M.S.; Grice, J.E.; Mohammed, Y. Quality by Design: Development of the Quality Target Product Profile (QTPP) for Semisolid Topical Products. Pharmaceutics 2020, 12, 287. [CrossRef] [PubMed]

24. Shirsat, A.E.; Chitlange, S.S. Application of quality by design approach to optimize process and formulation parameters of rizatriptan loaded chitosan nanoparticles. J. Adv. Pharm. Technol. Res. 2015, 6, 88-96. [CrossRef] [PubMed] 
25. Barakat, N.S. Optimization of physical characterization, skin permeation of naproxen from glycofurol-based topical gel. Asian J. Pharm. 2014, 4. [CrossRef]

26. Sharma, A.; Pahwa, S.; Bhati, S.; Kudeshia, P. Spanlastics: A modern approach for nanovesicular drug delivery system. Int. J. Pharm. Sci. Res. 2020, 11, 1057-1065. [CrossRef]

27. Ranade, S.; Thiagarajan, P. Selection of a design for response surface. IOP Conf. Ser. Mater. Sci. Eng. 2017, 263, 022043. [CrossRef]

28. Elsenosy, F.M.; Abdelbary, G.A.; Elshafeey, A.H.; Elsayed, I.; Fares, A.R. Brain Targeting of Duloxetine HCL via Intranasal Delivery of Loaded Cubosomal Gel: In Vitro Characterization, ex vivo Permeation, and in vivo Biodistribution Studies. Int. J. Nanomed. 2020, 15, 9517-9537. [CrossRef]

29. Yoshioka, T.; Sternberg, B.; Florence, A.T. Preparation and properties of vesicles (niosomes) of sorbitan monoesters (Span 20, 40, 60 and 80) and a sorbitan triester (Span 85). Int. J. Pharm. 1994, 105, 1-6. [CrossRef]

30. Al-Mahallawi, A.M.; Abdelbary, A.A.; Aburahma, M.H. Investigating the potential of employing bilosomes as a novel vesicular carrier for transdermal delivery of tenoxicam. Int. J. Pharm. 2015, 485, 329-340. [CrossRef]

31. Fahmy, A.M.; El-Setouhy, D.A.; Habib, B.A.; Tayel, S.A. Enhancement of Transdermal Delivery of Haloperidol via Spanlastic Dispersions: Entrapment Efficiency vs. Particle Size. AAPS PharmSciTech 2019, 20, 95. [CrossRef]

32. Shilo, M.; Sharon, A.; Baranes, K.; Motiei, M.; Lellouche, J.-P.M.; Popovtzer, R. The effect of nanoparticle size on the probability to cross the blood-brain barrier: An in-vitro endothelial cell model. J. Nanobiotechnol. 2015, 13, 19. [CrossRef] [PubMed]

33. Mazyed, E.; Helal, D.; Elkhoudary, M.; Elhameed, A.A.; Yasser, M. Formulation and Optimization of Nanospanlastics for Improving the Bioavailability of Green Tea Epigallocatechin Gallate. Pharmaceuticals 2021, 14, 68. [CrossRef] [PubMed]

34. Mehanna, M.M.; Motawaa, A.M.; Samaha, M.W. Nanovesicular carrier-mediated transdermal delivery of tadalafil: I-Formulation and physicsochemical characterization. Drug Dev. Ind. Pharm. 2015, 41, 714-721. [CrossRef] [PubMed]

35. Kamboj, S.; Saini, V.; Bala, S. Formulation and Characterization of Drug Loaded Nonionic Surfactant Vesicles (Niosomes) for Oral Bioavailability Enhancement. Sci. World J. 2014, 2014, 959741. [CrossRef]

36. Wang, N.; Hsu, C.; Zhu, L.; Tseng, S.; Hsu, J.-P. Influence of metal oxide nanoparticles concentration on their zeta potential. J. Colloid Interface Sci. 2013, 407, 22-28. [CrossRef]

37. Junyaprasert, V.B.; Teeranachaideekul, V.; Supaperm, T. Effect of Charged and Non-ionic Membrane Additives on Physicochemical Properties and Stability of Niosomes. AAPS PharmSciTech 2008, 9, 851-859. [CrossRef]

38. Zhao, Y.; Wang, Y.; Ran, F.; Cui, Y.; Liu, C.; Zhao, Q.; Gao, Y.; Wang, D.; Wang, S. A comparison between sphere and rod nanoparticles regarding their in vivo biological behavior and pharmacokinetics. Sci. Rep. 2017, 7, 4131. [CrossRef]

39. Vyas, T.K.; Shahiwala, A.; Marathe, S.; Misra, A. Intranasal Drug Delivery for Brain Targeting. Curr. Drug Deliv. 2005, 2, 165-175. [CrossRef]

40. Kreuter, J.; Shamenkov, D.; Petrov, V.; Ramge, P.; Cychutek, K.; Koch-Brandt, C.; Alyautdin, R. Apolipoprotein-mediated Transport of Nanoparticle-bound Drugs across the Blood-Brain Barrier. J. Drug Target. 2002, 10, 317-325. [CrossRef]

41. El Zaafarany, G.M.; Awad, G.A.; Holayel, S.M.; Mortada, N.D. Role of edge activators and surface charge in developing ultradeformable vesicles with enhanced skin delivery. Int. J. Pharm. 2010, 397, 164-172. [CrossRef] [PubMed]

42. Agrawal, R.; Sandhu, S.K.; Sharma, I.; Kaur, I.P. Development and Evaluation of Curcumin-loaded Elastic Vesicles as an Effective Topical Anti-inflammatory Formulation. AAPS PharmSciTech 2015, 16, 364-374. [CrossRef] [PubMed]

43. Ioele, G.; Tavano, L.; De Luca, M.; Ragno, G.; Picci, N.; Muzzalupo, R. Photostability and ex-vivo permeation studies on diclofenac in topical niosomal formulations. Int. J. Pharm. 2015, 494, 490-497. [CrossRef] [PubMed] 